\title{
Inverse Estimation Method of Material Randomness Using Observation
}

\author{
Dae-Young Kim ${ }^{1}$, Pawel Sikora ${ }^{2,3}$, , Krystyna Araszkiewicz $^{3}{ }^{-1}$ and Sang-Yeop Chung ${ }^{1, *}$ \\ 1 Department of Civil and Environmental Engineering, Sejong University, 209 Neungdong-ro, Gwangjin-gu, \\ Seoul 05006, Korea; kd2young@gmail.com \\ 2 Building Materials and Construction Chemistry, Technische Universität Berlin, Gustav-Meyer-Allee 25, \\ 13355 Berlin, Germany; pawel.sikora@zut.edu.pl \\ 3 Faculty of Civil Engineering and Architecture, West Pomeranian University of Technology Szczecin, Al. \\ Piastow 50, 70-311 Szczecin, Poland; krystyna.araszkiewicz@zut.edu.pl \\ * Correspondence: sychung@sejong.ac.kr; Tel.: +82-2-6935-2471
}

Received: 16 April 2020; Accepted: 15 June 2020; Published: 16 June 2020

check for updates

\begin{abstract}
This study proposes a method for inversely estimating the spatial distribution characteristic of a material's elastic modulus using the measured value of the observation data and the distance between the measurement points. The structural factors in the structural system possess temporal and spatial randomness. One of the representative structural factors, the material's elastic modulus, possesses temporal and spatial randomness in the stiffness of the plate structure. The structural factors with randomness are typically modeled as having a certain probability distribution (probability density function) and a probability characteristic (mean and standard deviation). However, this method does not consider spatial randomness. Even if considered, the existing method presents limitations because it does not know the randomness of the actual material. To overcome the limitations, we propose a method to numerically define the spatial randomness of the material's elastic modulus and confirm factors such as response variability and response variance.
\end{abstract}

Keywords: Bayesian updating; spatial randomness; uncertainty; correlation distance; stochastic field

\section{Introduction}

Research toward the development and incorporation of new building materials in modern engineering structures in order to meet sustainability goals has gathered substantial attention in recent years. Various new cement-based composites have been investigated including lightweight materials (foamed concrete and lightweight aggregates concretes) [1], pervious concretes [2], nano-modified, and self-cleaning materials $[3,4]$. However, due to the relatively higher production costs of modern building materials than in the case of conventional ones, it is still imperative to find a solution to support the simulating techniques and their accuracy toward decreasing the number of site trials, thus reducing the costs and environmental impact of material.

In general, structural material analysis can be classified into a deterministic or a probabilistic method [5]. The deterministic method uses finite element analysis (FEA) considering the material properties, geometry, and forces. In other words, the various internal and external factors that can exist in a structure are represented by constants. However, these factors are all assumptions, and in the case of actual structures, it would be more reasonable to assume that the factors have different values depending on the position vector in the structural domain.

The probabilistic method assumes that the structure possesses arbitrary material properties, loads, and geometries, and generates a random sample with some statistical characteristics. A deterministic FEA is repeatedly performed on the generated samples to obtain the characteristic behavior of 
the structure [6,7]. Structural uncertainty, in terms of numerical considerations, can be classified as intrinsic, measurement, and statistical uncertainty, or uncertainty in the mathematical model [8,9]. In each case, the measurement uncertainty is that involved in establishing uncertainty factors through experiments. Experimental and statistical uncertainty is that obtained due to the lack of data due to limited time and space information. Uncertainty in the mathematical model implies uncertainty due to the difference between the actual model of the uncertainty coefficient and the simulated mathematical model. Intrinsic uncertainty is an uncertainty in the structural material, geometric factors expressing the shape of structures, and applied loads $[10,11]$. These uncertainties are generally considered, both in the actual behavior of the structure and in the reliability analysis. Among them, the stochastic finite element method (SFEM) is mainly focused on the intrinsic uncertainty with the greatest influence. SFEM is a combination of a stochastic method and FEM. The purpose of SFEM is to estimate the uncertain response variation of the structure with respect to the spatial and temporal randomness of the factors in the structural system $[12,13]$. SFEM is divided into statistical and non-statistical methods in terms of analytical methodology.

There are many analytical methods such as the K-L Expansion [14], Polynomial Chaos Expansion [15], perturbation methods [16], and the weighted integration method that is based on non-statistical methods [17-22]. However, the non-statistical method is mainly based on the first-order expansion or the second-order series expansion for the main variables and is applicable only when the coefficient of variation (COV) of the stochastic fields is low [23]. In fact, when COV $(=\sigma / \mu)$ of the stochastic field is large, it is accurate and shows a significant difference from the Monte-Carlo simulation (MCS).

The MCS, which is a representative statistical method, has the advantage of providing a solution to most stochastic problems. However, to obtain a MCS with high efficiency and accuracy, a proper algorithm and considerable time is also required for analysis. This study focuses on estimating the spatial randomness of materials through observation (partial elastic modulus) and overcoming the limitations of the existing statistical methods. For this purpose, a method to numerically define the spatial randomness of the material's elastic modulus was proposed, and the obtained results were demonstrated using factors such as response variability and response variance.

\section{Disadvantage of Current Statistical Methods}

\subsection{Statistical Methods without Considering Spatial Randomness}

In the current statistical method, the target random variable, which is the elastic modulus, is assumed to be a normal distribution $(\mathrm{N}(\mu, \sigma))$. In this case, a random process satisfying a specific normal distribution is generated as a pseudo time process (here, the random variable satisfies $r_{t}=r(t), t \in(0, T)$ and an analysis is performed for generated $N$ constant to satisfy the normal distribution $(N(\mu, \sigma))$.

However, as shown in Figure 1, the factor of the actual material's elastic modulus does not have the same value according to the position vector. Therefore, the current method of using a constant field is different from the actual material, and a random sample of the statistical method should satisfy both functions simultaneously.

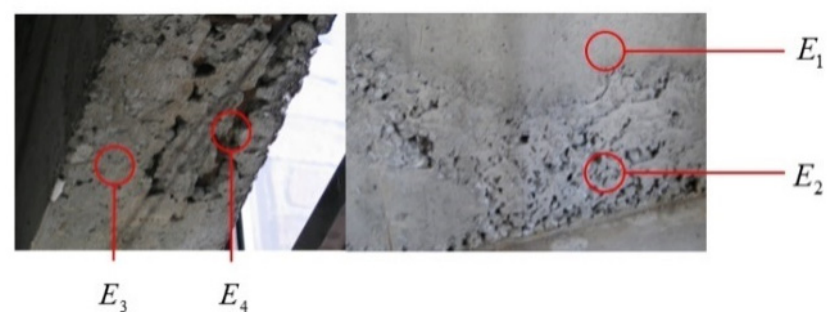

Figure 1. Different elastic modulus in different positions due to material heterogeneity. 


\subsection{Statistical Methods Considering Spatial Randomness}

The spatial randomness distribution of the uncertainty factor is represented by $f(x)$ in the stochastic field function, stochastic field $f\left(x_{i}\right)$, and at any point $x_{i} \in \Omega_{s t r}$, belonging to the structural domain $\Omega_{s t r}$, which must satisfy the probability density function (PDF). At the same time, it must also satisfy the spectral density function (SDF) (or autocorrelation function) representing the aspect existing in the structural domain [24]. Even in the case of a stochastic field with the same mean and standard deviation, the actual shape of the stochastic field can exist in various forms $\left(\mu_{i}^{k}=\mu_{j}^{k}\right)$. This variance is due to the characteristics of the stochastic field based on the ensemble concept, which is a statistical characteristic in a direction orthogonal to the stochastic plane.

In general, the distribution characteristic of the stochastic field is determined by the correlation distance. The stochastic field can have two extreme conditions: $f(x ; d=0)$ and $f(x ; d=\infty)$. In the former case, the stochastic field appears in the form of a white noise field and contains all the theoretically possible spectra. In the latter, the stochastic field is a constant field with one value, which is called the random variable state [25]. For example, $d$ is infinite when spatial randomness is not taken into consideration.

The distribution of the stochastic field using the correlation distance can simulate (imitate) the spatial randomness of the material. However, it is difficult to determine the spatial randomness of the actual material with this approach. Various methods are available to compute the elastic modulus of concrete, but the spatial randomness is difficult to identify. To investigate the spatial randomness of a target objective, the stochastic field with a correlation distance (d) ranging from zero to infinity needs to be generated. In addition, this spatial characteristic can be examined by the COV trend obtained from the Monte-Carlo simulation, as shown in Figure 2 [26,27].

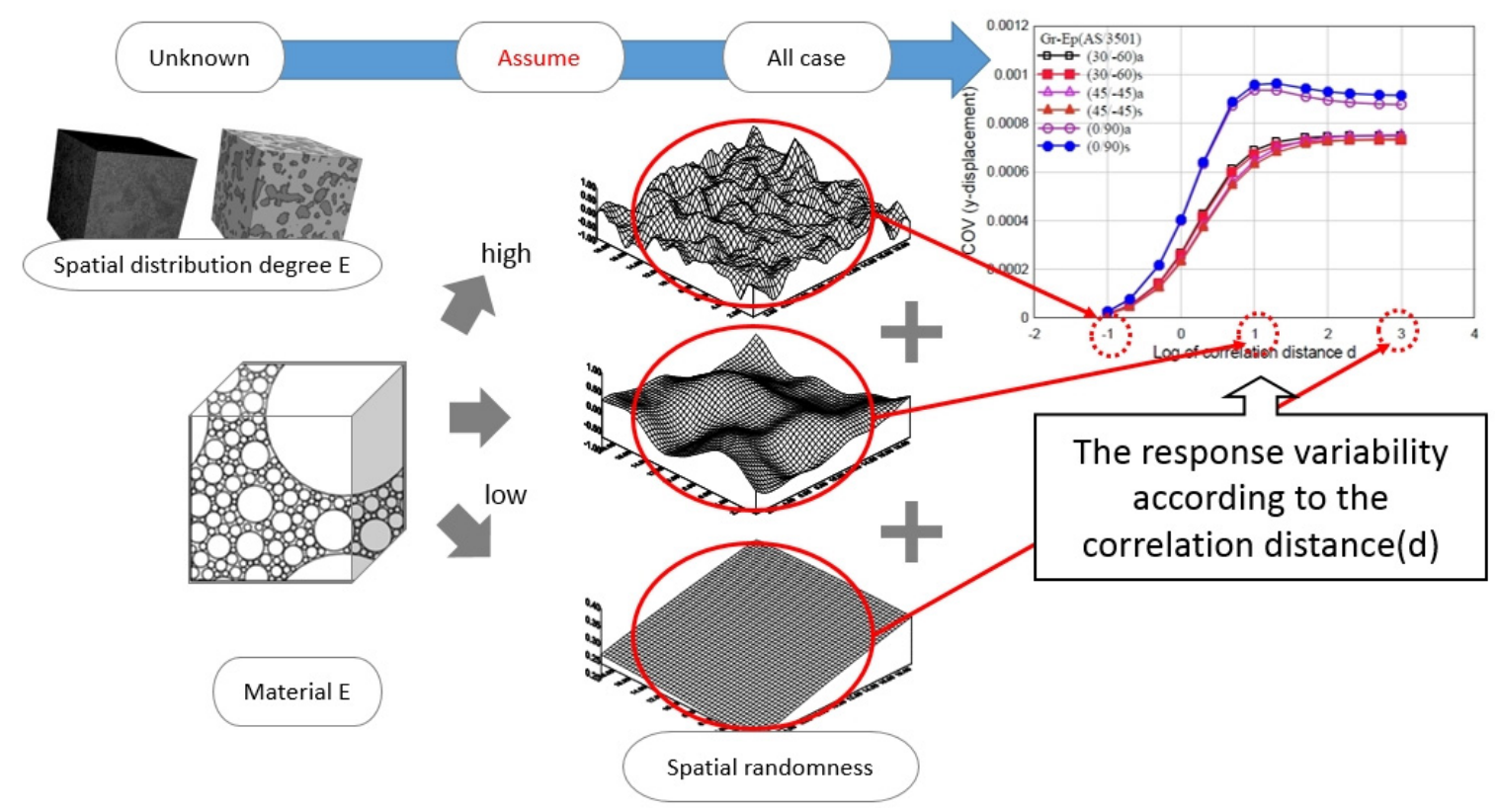

Figure 2. The schematic of the response variability according to the correlation distance (d).

However, since the actual correlation distance (d) of the target elastic modulus cannot be known, the exact result is unknown (which is not a meaningful result). Therefore, we aimed to overcome the problem (disadvantages) of the existing statistical methods.

\subsection{Objective and Methods}

In this study, we proposed a method of inversely estimating the correlation distance using the observed values and the distance between the observation points. In particular, the population can be predicted using some samples. Then, the obtained real correlation distance $d_{1}$ (from the population) 
is compared with the $\mathrm{d}_{2}$ (predicted from samples). Once the correlation distance of the actual material is found using the proposed method, the exact behavior of structures by the actual material can be described instead of using the tendency of COV. The process is described in the following three steps. First, generate the stochastic field using the spectral representation method $[28,29]$. The purpose of creating a sample by applying the algorithm is because the exact $d$ value corresponding to the samples through the algorithm is known. If the proposed inverse estimation method correctly traces $d$, it starts from the assumption that some data of the actual structure can be used to obtain an actual $\mathrm{d}$ corresponding to the structure. Second, we assumed values at some locations to be used as sample data, as shown in Figure 3, and compared the estimated correlation distance (using only some observation) and the correlation distance of the stochastic field. Third, the probability characteristics are obtained using the Bayesian method. This is a method of obtaining new probability characteristics (posterior) by updating the prior probability characteristics based on new test data [30].

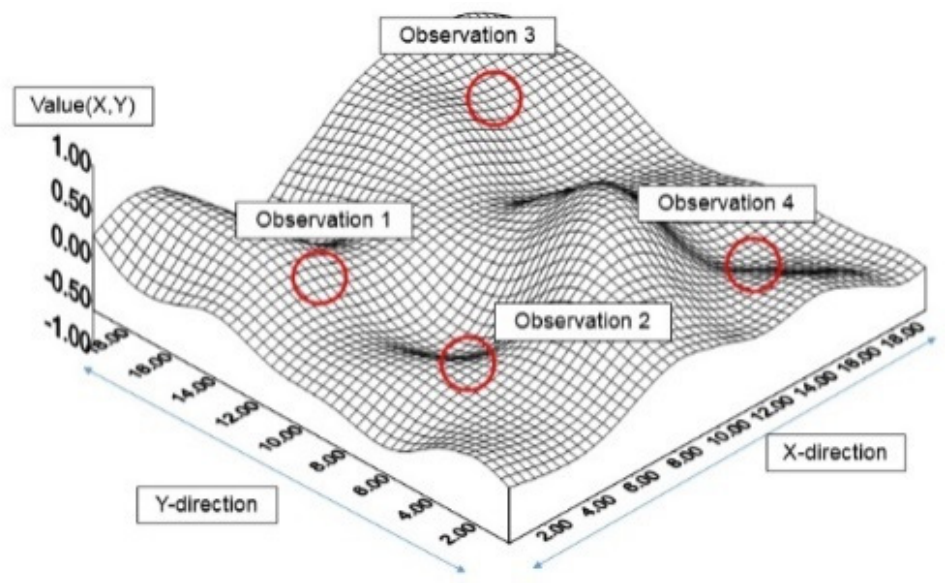

Figure 3. Observation in the stochastic field with spatial randomness.

\section{Investigation Procedures and Their Examples}

\subsection{Consideration Method of Spatial Randomness of Material's Elastic Modulus}

For every real-valued $2 \mathrm{D}-1 \mathrm{~V}$ homogeneous stochastic field $f_{0}\left(x_{1}, x_{2}\right)$ with the mean value equal to zero and a bi-quadrant SDF as $G_{f_{0} f_{0}}\left(\kappa_{1}, \kappa_{2}\right)$, two mutually orthogonal real processes $u\left(\kappa_{1}, \kappa_{2}\right)$ and $v\left(\kappa_{1}, \kappa_{2}\right)$ with orthogonal increments $d u\left(\kappa_{1}, \kappa_{2}\right)$ and $d v\left(\kappa_{1}, \kappa_{2}\right)$ can be assigned so that:

$$
\begin{gathered}
f_{0}\left(x_{1}, x_{2}\right)=\int_{-\infty}^{\infty}\left[\cos \left(\kappa_{1} x_{1}+\kappa_{2} x_{2}\right) d u\left(\kappa_{1}, \kappa_{2}\right)+\sin \left(\kappa_{1} x_{1}+\kappa_{2} x_{2}\right) d v\left(\kappa_{1}, \kappa_{2}\right)\right] \\
E(x, y)=\bar{E}\left(1+f_{i}(x, y)\right) \\
R\left(\xi_{1}, \xi_{2}\right)=\sigma_{0}^{2} \exp \left\{-\left(\frac{\Delta \xi_{1}}{d_{1}}\right)-\left(\frac{\Delta \xi_{2}}{d_{2}}\right)\right\}
\end{gathered}
$$

In the generation of homogeneous random fields, the spectral representation method [31-33] can be employed, which takes advantage of fast Fourier transform. In the random fields of elastic modulus, the stiffness of the elastic modulus is assumed to be homogeneous Gaussian and represented by Equation (2), where $\bar{E}$ is the mean value of the elastic modulus and $f_{i}(x, y)$ is a homogeneous random field. The auto-correlation functions for the respective random field $f_{i}(x, y)$ are assumed by Equation (3) [34]. In the spectral representation scheme, the numerical generation of a homogeneous 
uni-variate i-th random sample $f_{i}(x, y)$ with a zero mean in two dimensions can be generated via the summation of the cosine functions, as shown in Equation (4) [35,36].

$$
\begin{gathered}
f_{i}(x, y)=\sqrt{2} \sum_{n 1=0}^{N_{1}-1} \sum_{n 1=0}^{N_{2}-1}\left[A_{n 1 n 2} \cos \left(\kappa_{1 n_{1}} x+\kappa_{2 n_{2}} y+\Phi_{n_{1} n_{2}}^{(1)(i)}\right)\right. \\
\left.+\widetilde{A}_{n 1 n 2} \cos \left(\kappa_{1 n_{1}} x-\kappa_{2 n_{2}} y+\Phi_{n_{1} n_{2}}^{(2)(i)}\right)\right] \\
A_{n 1 n 2}=\sqrt{2 S_{f_{0} f_{0}}\left(\kappa_{1 n_{1}}, \kappa_{2 n_{2}}\right) \Delta \kappa_{1} \Delta \kappa_{2},} \widetilde{A}_{n 1 n 2}=\sqrt{2 S_{f_{0} f_{0}}\left(\kappa_{1 n_{1}},-\kappa_{2 n_{2}}\right) \Delta \kappa_{1} \Delta \kappa_{2}} \\
\kappa_{1 n_{1}}=n_{1} \Delta \kappa_{1} ; \quad \kappa_{2 n_{2}}=n_{2} \Delta \kappa_{2} \quad \Delta \kappa_{1}=\kappa_{1 u} / N_{1} ; \quad \Delta \kappa_{2}=\kappa_{2 u} / N_{2}
\end{gathered}
$$

Since the uniform random phase angle $\Phi_{n_{1} n_{2}}$ in Equation (4) is determined depending on $n_{1}$ and $n_{2}$, it needs to generate two folds of $N_{1} \times N_{2}$ number of values in the range of $[0,2 \pi]$. The upper cut-off limit of wave numbers $\kappa_{1 u}, \kappa_{2 u}$ in the SDF $S_{f 0 f 0}\left(\kappa_{1}, \kappa_{1}\right)$ can be determined by:

$$
\int_{0}^{\kappa_{1} u} \int_{-\kappa_{2} u}^{\kappa_{2} u} S_{f_{0} f_{0}}\left(\kappa_{1}, \kappa_{2}\right) d \kappa_{1} d \kappa_{2}=(1-\varepsilon) \int_{0}^{\infty} \int_{-\infty}^{\infty} S_{f_{0} f_{0}}\left(\kappa_{1}, \kappa_{2}\right) d \kappa_{1} d \kappa_{2}
$$

where $\varepsilon$ is set to be $0.001(0.1 \%)$ in the numerical generation.

In the basic spectral representation method, as shown in Figure 4, 2D-1V can be expressed as a stochastic field, depending on the mean, standard deviation, and the correlation distance between variables [37].

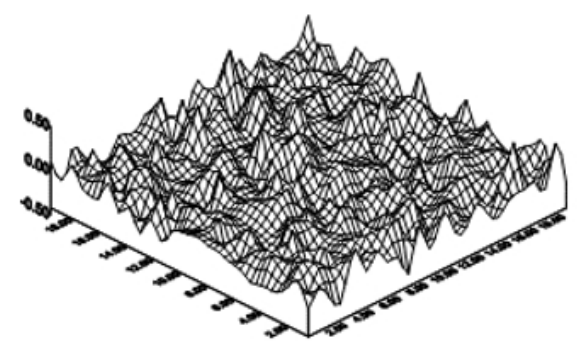

(a)

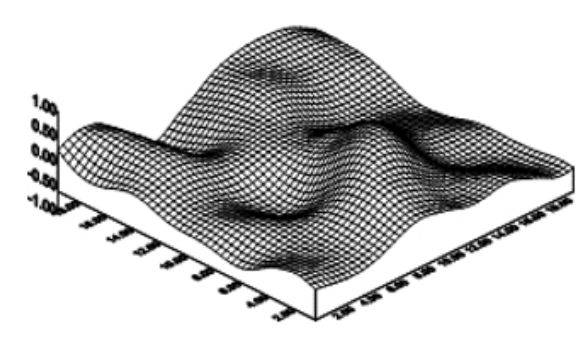

(c)

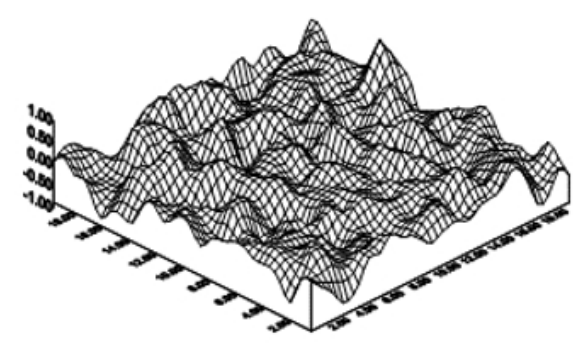

(b)

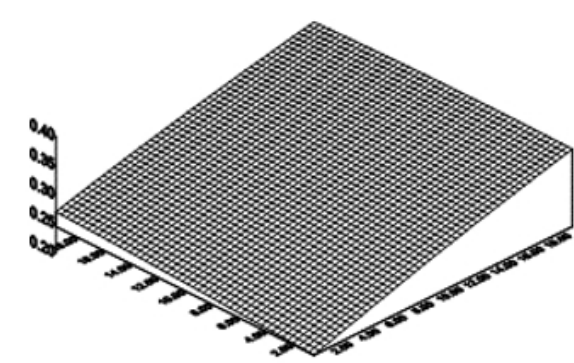

(d)

Figure 4. Stochastic field (2D-1V): (a) $d=1.0 ;(\mathbf{b}) d=5.0 ;(\mathbf{c}) d=10.0 ;(d) d=50.0$.

\subsection{Bayesian Method}

Bayesian inference is a method of statistical inference in which Bayes' theorem is used to update the probability for a hypothesis as more evidence or information becomes available. Bayesian inference is an important technique in statistics, especially in mathematical statistics. Bayesian updating is particularly important in the dynamic analysis of a sequence of data. 
Bayesian inference derives the posterior probability as a consequence of two antecedents: a prior probability and a "likelihood function" derived from a statistical model for the observed data. Bayesian inference computes the posterior probability according to Bayes' theorem:

$$
\begin{aligned}
& \text { posterior } \propto \text { likelihood } \times \text { prior } \\
& p(\theta \mid \text { data }) \propto p(\text { data } \mid \theta) \times p(\theta)
\end{aligned}
$$

where $\theta$ stands for any hypothesis whose probability may be affected by data (called evidence below) [38,39]. Often, there are competing hypotheses, and the task is to determine which is the most probable. $p(\theta)$, the prior probability, is the estimate of the probability of the hypothesis $\theta$ before the "data," the current evidence, is observed. The evidence "data" corresponds to new data that was not used in computing the prior probability.

$p(\theta \mid$ data $)$, the posterior probability, is the probability of $\theta$ given "data", after the "data" are observed. This is what we want to know: the probability of a hypothesis given the observed evidence. $p($ data $\mid \theta)$ is the probability of observing "data" given $\theta$, and is called the likelihood. The likelihood function is a function of the evidence, data, while the posterior probability is a function of the hypothesis, $\theta$ and can be expressed by the process as shown in Figure 3 [40].

$$
\begin{gathered}
p\left(\mu, \sigma^{2}\right) \propto \sigma^{-1}\left(\sigma^{2}\right)^{-\left(v_{0} / 2+1\right)} \exp \left[-\frac{1}{2 \sigma^{2}}\left\{v_{0} \sigma_{0}^{2}+\kappa_{0}\left(\mu-\mu_{0}\right)^{2}\right\}\right] \\
\times\left(\sigma^{2}\right)^{-n / 2} \exp \left[-\frac{1}{2 \sigma^{2}}\left\{(n-1) s^{2}+n(\bar{y}-\mu)^{2}\right\}\right] \\
\mu_{n}=\frac{\kappa_{0}}{\kappa_{0}+n} \mu_{0}+\frac{n}{\kappa_{0}+n} \bar{y} \\
\kappa_{n}=\kappa_{0}+n ; v_{n}=v_{0}+n ; \quad \kappa_{0}=s^{2} / \sigma_{0}^{2} \\
v_{n} \sigma_{n}^{2}=v_{0} \sigma_{0}^{2}+(n-1) s^{2}+\frac{\kappa_{0} n}{\kappa_{0}+n}\left(\bar{y}-\mu_{0}\right)^{2}
\end{gathered}
$$

It is expressed as the product of likelihood and prior probability if expanded to a probability distribution, as shown in Equation (10) (posterior probability) [41]. Here, $\mu_{0}, \mu_{n}$ denotes the prior/posterior mean, $\sigma_{0}, \sigma_{n}$ denotes the prior/posterior standard deviation, and $v_{0}, v_{n}$ denotes the prior/posterior degree of freedom. Similarly, $\bar{y}, s$, and $n$ are the test mean, test standard deviation, and test count, respectively, where $s^{2}=\left(\left(y_{1}-\bar{y}\right)^{2}+\cdots+\left(y_{n}-\bar{y}\right)^{2}\right) / n$.

\subsection{Consideration of Inverse Estimation (Prediction) Method}

For visualization purposes, we considered only 1-axis direction ( $\mathrm{y}=$ constant or $\mathrm{x}=$ constant $)$ of the 2D-1V stochastic field $f_{0}(x)$, as shown in Figure $5 \mathrm{a}$. The method of estimating the correlation distance, expressing the spatial distribution state, is as follows [42]. First, within the stochastic field, each random variable has an irregular value. Second, the difference in value between two consecutive random variables also has an irregular value, but when $d=1$, it will be larger than $d=10$. Similarly, as shown in Figure 5c, it is assumed that the non-continuous random variables are also the same. Third, if the two random variables are equal in length, the maximum difference of the value will be similar in the stochastic field, regardless of the position. 


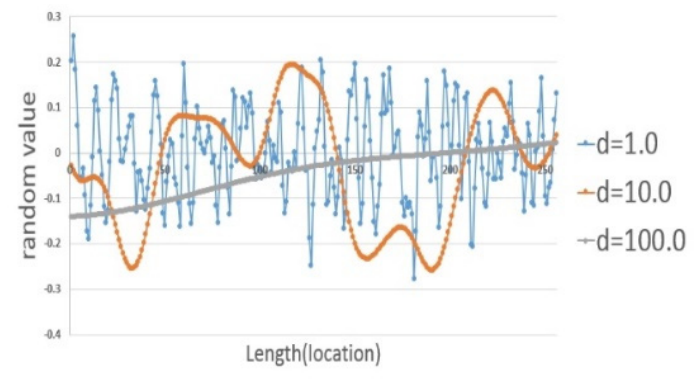

(a)

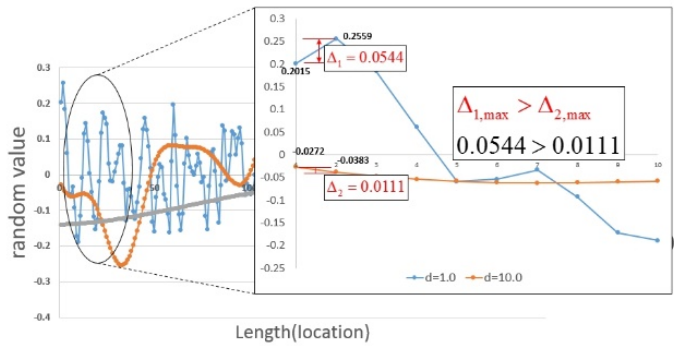

(b)

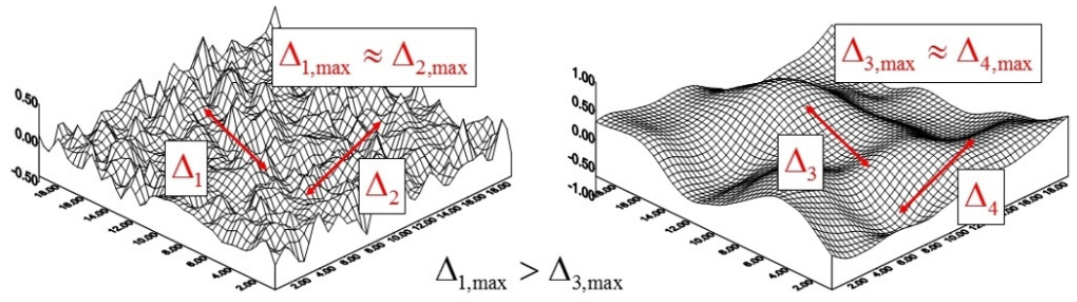

(d)

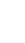

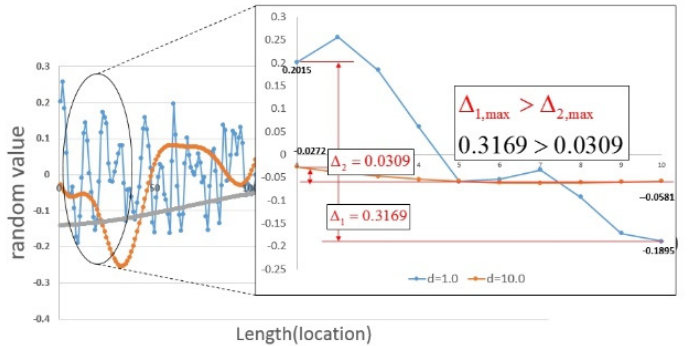

(c)

Figure 5. Difference of value between random variables: (a) Stochastic fields (1D-1V); (b) Difference of value in two continuous variables; (c) Difference of value in two non-continuous variables; (d) Comparison in stochastic fields with different correlation distance.

\section{Verification of the Proposed Method}

\subsection{Example Model and Method}

In this study, the main objective was to find the coefficient $(=\mathrm{d})$ representing the spatial randomness of the elastic modulus using experimental data (=observation) obtained by measuring specific parts of actual samples. However, it is not easy to accurately determine the spatial randomness of material through the measurement of the actual material. This is because there is almost no observed data of the actual structure. Therefore, as mentioned in Section 2, the population was predicted using some samples. Then, we compared the obtained real correlation distance $d_{1}$ from the population with the $d_{2}$ predicted from samples. We simulated the samples through an algorithm that knows the correlation distance $(\mathrm{d})$ value correctly. The process is described below.

First, as shown in Figure 6, 3000 stochastic fields are generated. Then, 100 out of the 3000 stochastic fields are selected, and the user arbitrarily determines a specific position of the stochastic field, and random variables at that position are collected. At this time, it is assumed that generated stochastic fields (of 3000) are the population measured using a relatively small number of samples (100 samples in this study). In addition, the collected data are assumed as the observation data. Third, the correlation distance is estimated using the collection of data and the Bayesian method; and the statistical characteristics $(\mu, \sigma)$ are obtained. Finally, the statistical characteristics of 3000 stochastic 
fields are compared with the updated statistical characteristics (and to compare the actual field $\mathrm{d}_{1}$ with estimated $\left.d_{2}\right)$. To verify the performance of the prediction, five cases with different conditions $(d=1,5$, $10,20,50)$ were selected and examined.

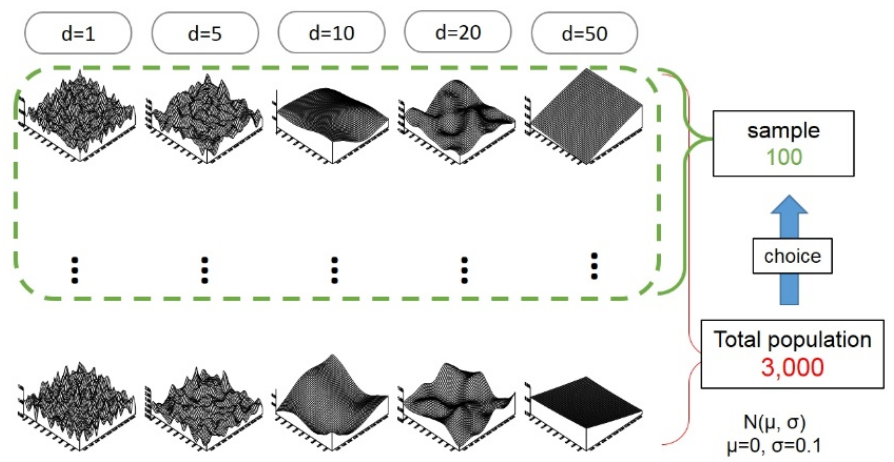

Figure 6. Procedures for estimation (sampling method).

The spatial randomness of the elastic modulus of the target material can be expressed by Equation (2) by applying the spectral representation method to generate stochastic fields $f_{i}(x, y)$ of $2 \mathrm{D}-1 \mathrm{~V}$. Here, it was assumed that $f_{i}(x, y)$ had a mean value of 0 , a standard deviation of 0.1 , and the probability density was assumed to be a normal distribution. $\bar{E}(=1.0)$ denotes the mean modulus of elasticity and $i$ (1 3000) denotes the number of stochastic fields. Then, we assumed the randomness of the elastic modulus using Equation (2).

The total number of element e of sample fields was 60 elements in the x-direction and 20 elements in the y-direction, as shown in Figure 7a However, we did not know the number of elements of actual material. Therefore, the correlation distance could be found without knowing the number of elements as shown in Figure 7b, Table 1, summarizes the measured values in Figure 7a.

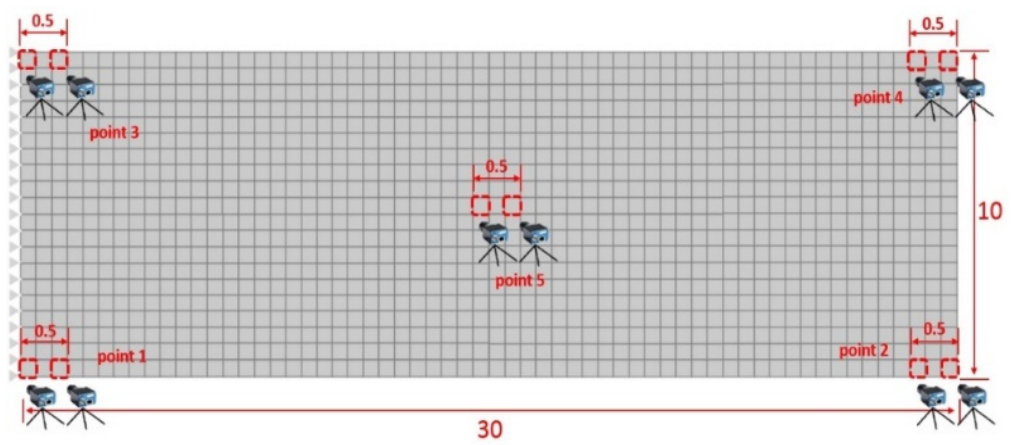

(a)

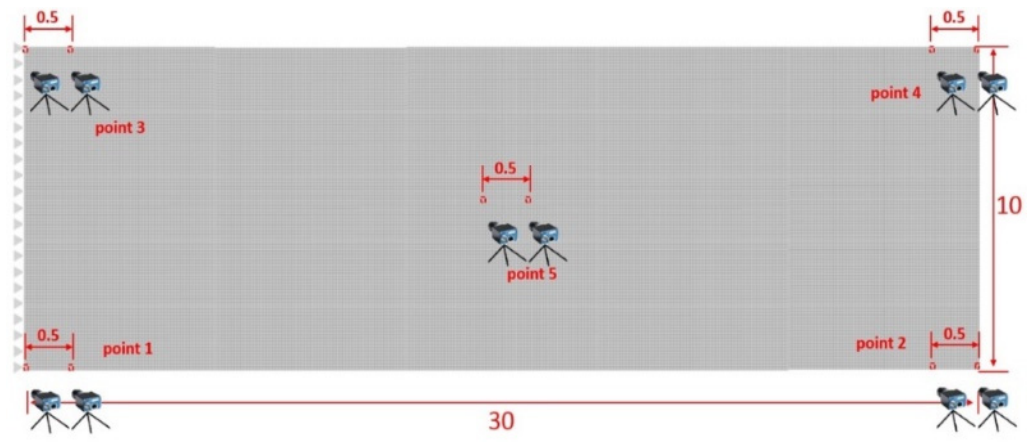

(b)

Figure 7. Design domain elements: (a) actual material 1200 elements; (b) estimated material 6000 elements. 
Table 1. Observation data at the distance $(=0.5)$ per correlation distance.

\begin{tabular}{cccccc}
\hline $\mathbf{M a x}\left(\mathbf{f}_{\mathbf{i}}\left(\mathbf{x}_{\mathbf{2}}\right)-\mathbf{f}_{\mathbf{i}}\left(\mathbf{x}_{\mathbf{1}}\right)\right)$ & $\mathbf{d}=\mathbf{1}$ & $\mathbf{d}=\mathbf{5}$ & $\mathbf{d}=\mathbf{1 0}$ & $\mathbf{d}=\mathbf{2 0}$ & $\mathbf{d}=\mathbf{5 0}$ \\
\hline Point 1 & 0.2211 & 0.0599 & 0.0282 & 0.0119 & 0.0046 \\
Point 2 & 0.1745 & 0.0640 & 0.0271 & 0.0117 & 0.0047 \\
Point 3 & 0.2241 & 0.0488 & 0.0282 & 0.0118 & 0.0055 \\
Point 4 & 0.1631 & 0.0442 & 0.0277 & 0.0116 & 0.0055 \\
Point 5 & 0.1832 & 0.0543 & 0.0275 & 0.0117 & 0.0050 \\
\hline
\end{tabular}

\subsection{Results}

Part (a) in Figures 8-12 are the stochastic fields composed of material homogeneity $(d=1,5,10$, $20,50)$. These five observation points are assumed to be material samples. The total population was assumed to be 3000, and the design was made so that the true value (elastic modulus) had a mean of 1.0 and a standard deviation of 0.1 . The prior probability was assumed to be unknown, with a mean of 0.8 and a standard deviation of 0.2 . A total of 100 out of 3000 stochastic fields were chosen continuously from arbitrary positions, and a Bayesian update of five observation points in each stochastic field was performed.

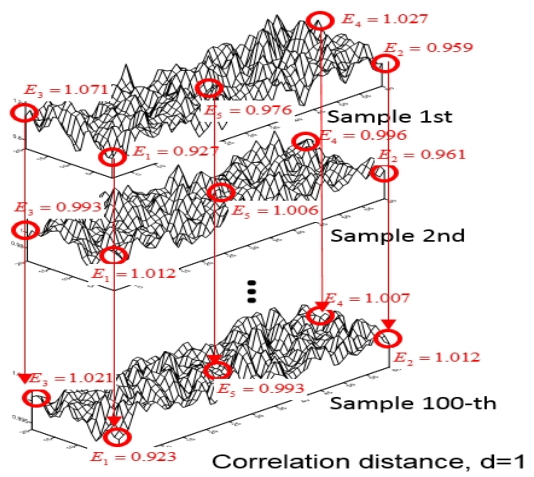

(a)

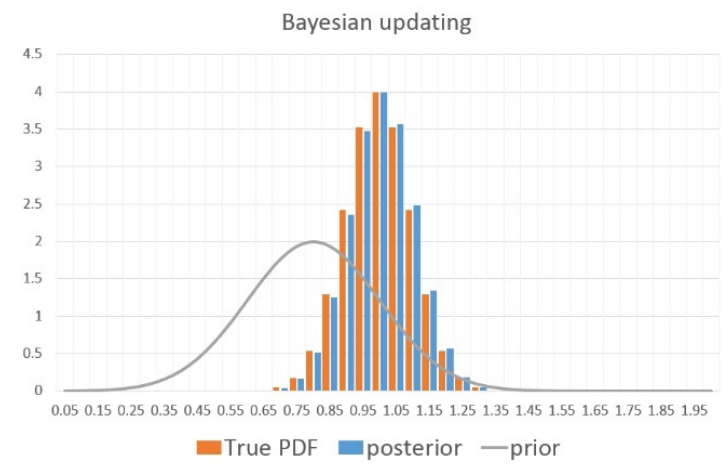

(c)

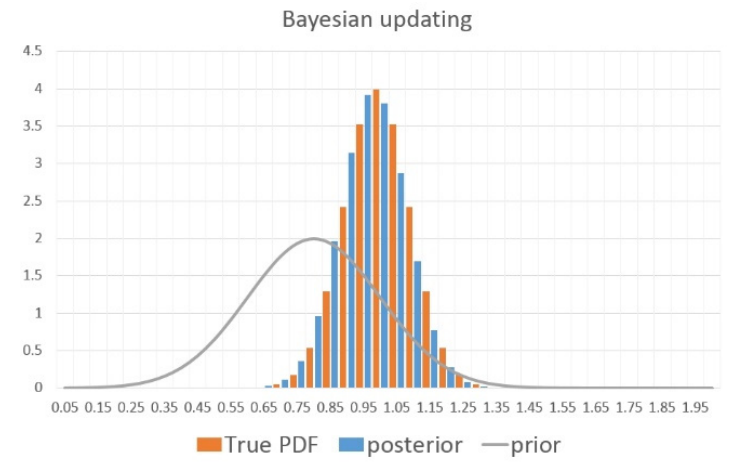

(b)

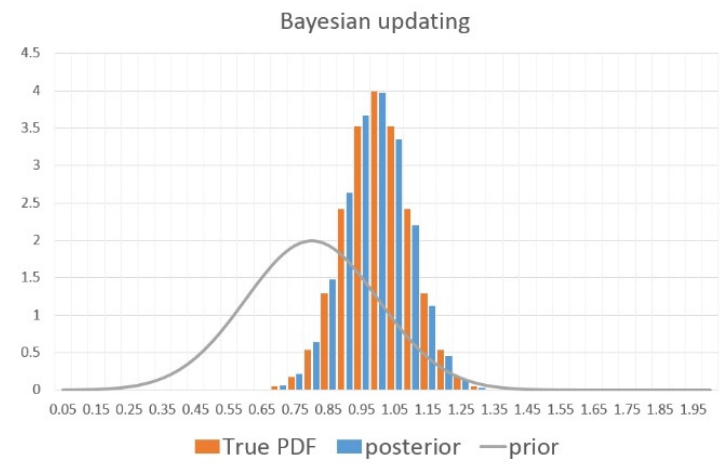

(d)

Figure 8. Cont. 


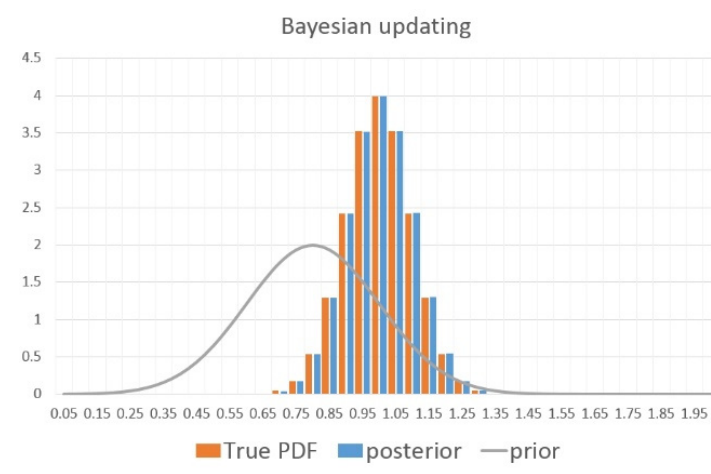

(e)

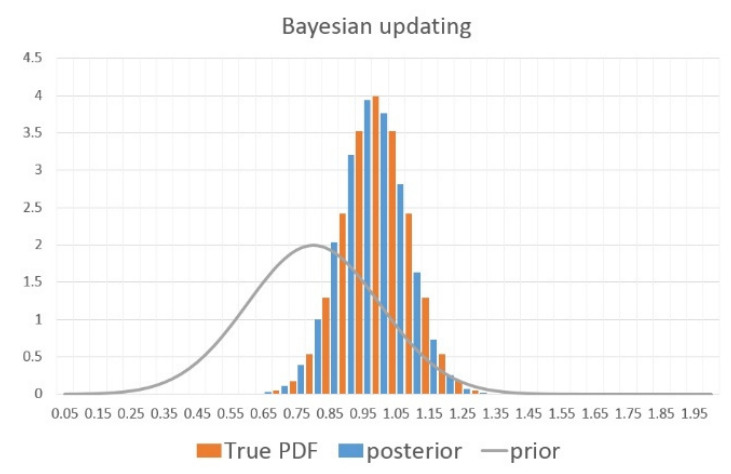

(f)

Figure 8. Bayesian updating at the stochastic field of the correlation distance $d=1.0$ : (a) How to perform Bayesian updating; (b) Observation point 1; (c) Observation point 2; (d) Observation point 3; (e) Observation point 4; (f) Observation point 5.

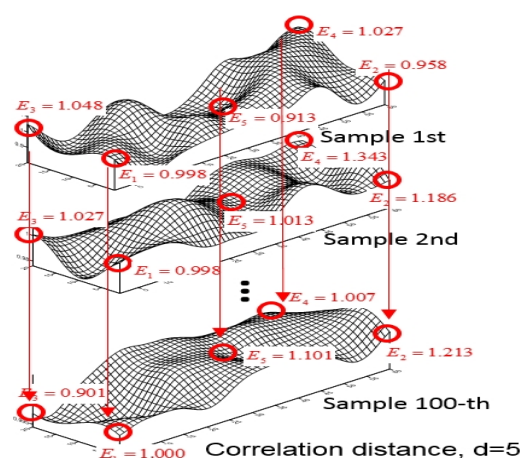

(a)

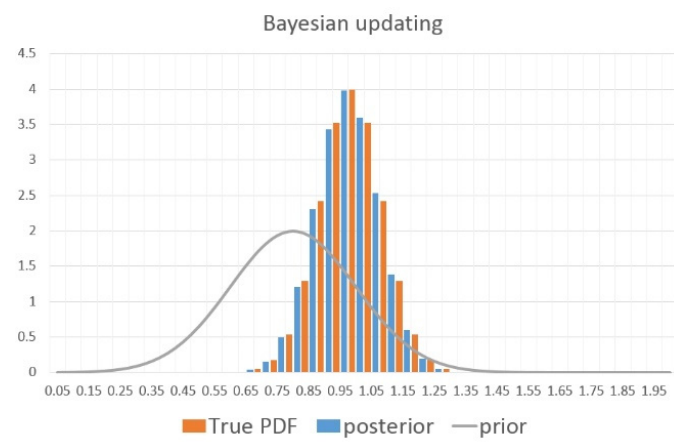

(c)

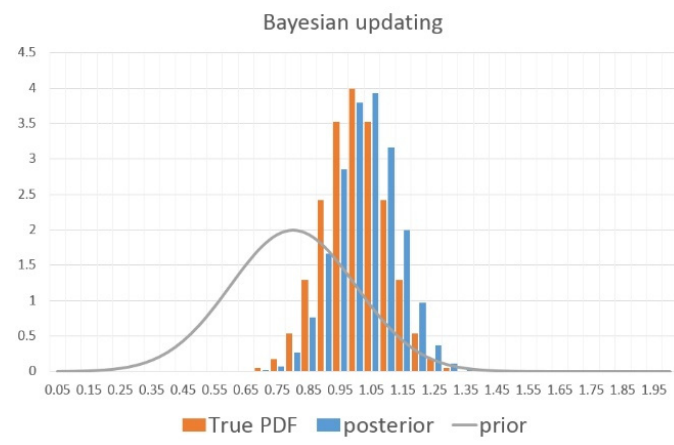

(e)

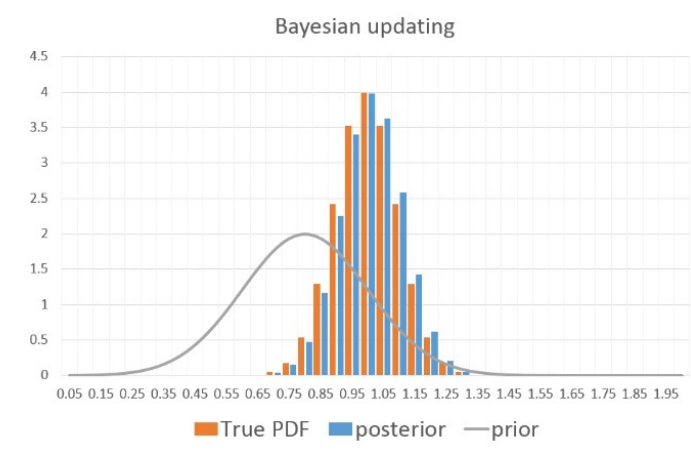

(b)

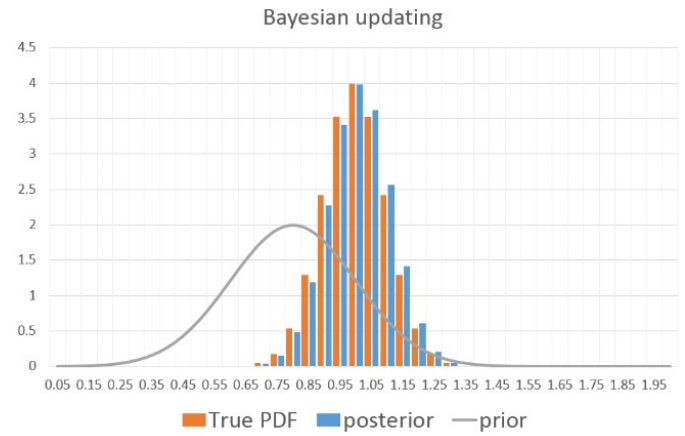

(d)

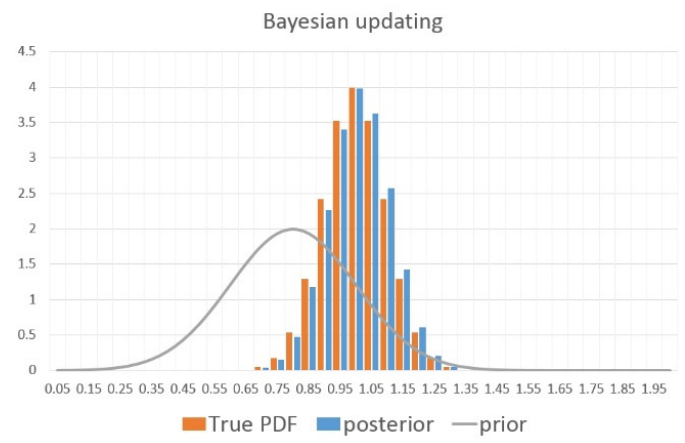

(f)

Figure 9. Bayesian updating at the stochastic field of the correlation distance $d=5.0$ : (a) How to perform Bayesian updating; (b) Observation point 1; (c) Observation point 2; (d) Observation point 3; (e) Observation point 4; (f) Observation point 5. 


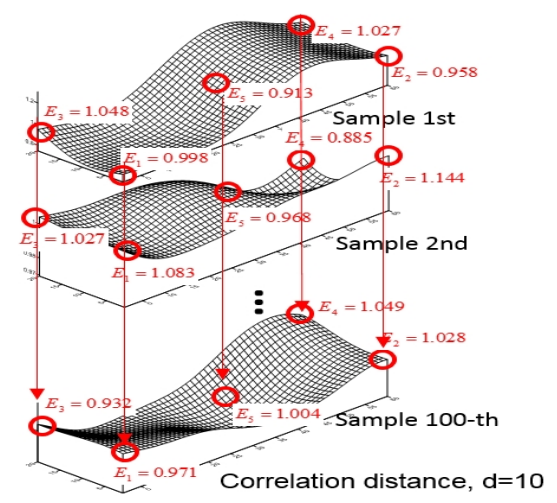

(a)

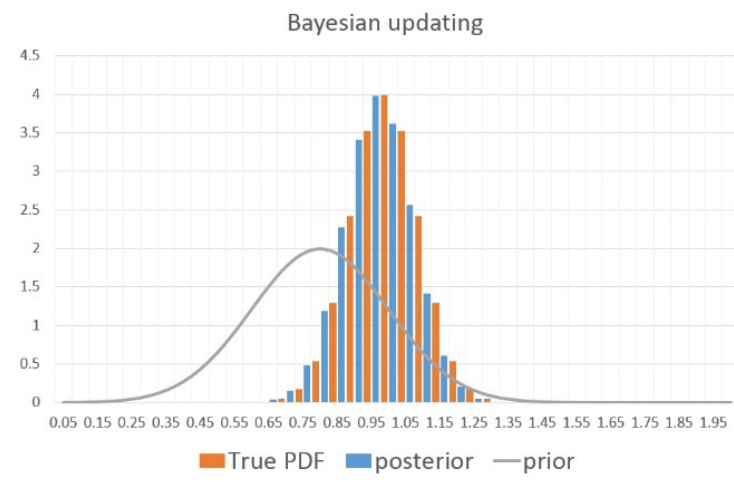

(c)

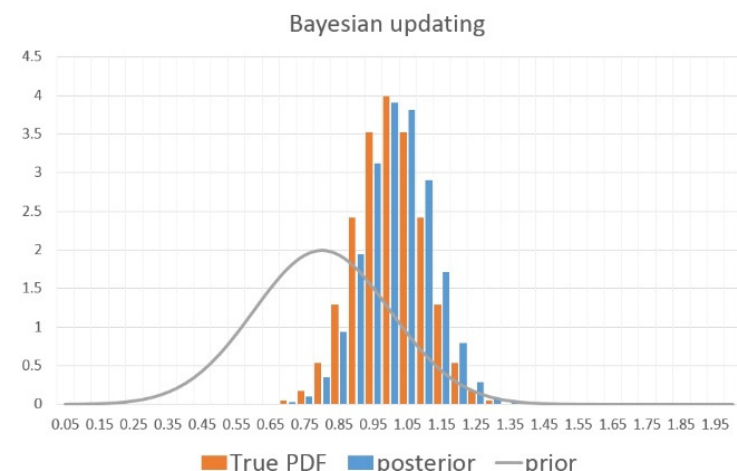

(e)

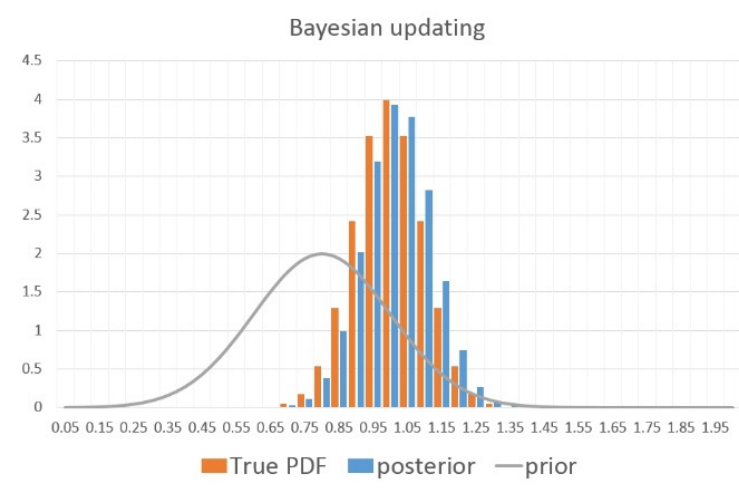

(b)

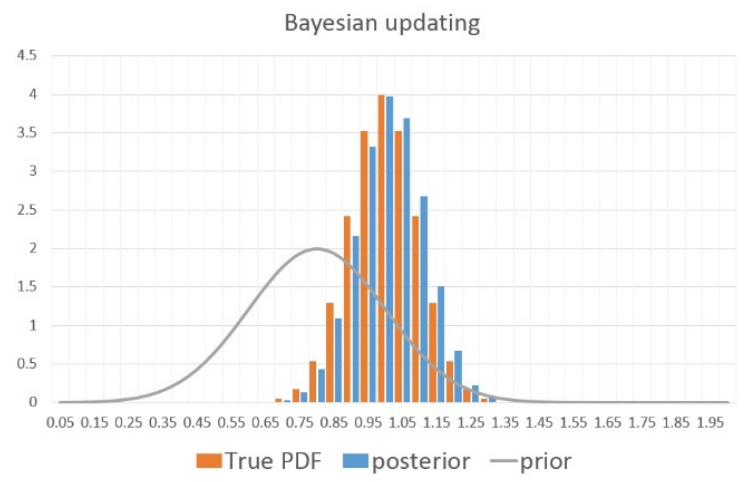

(d)

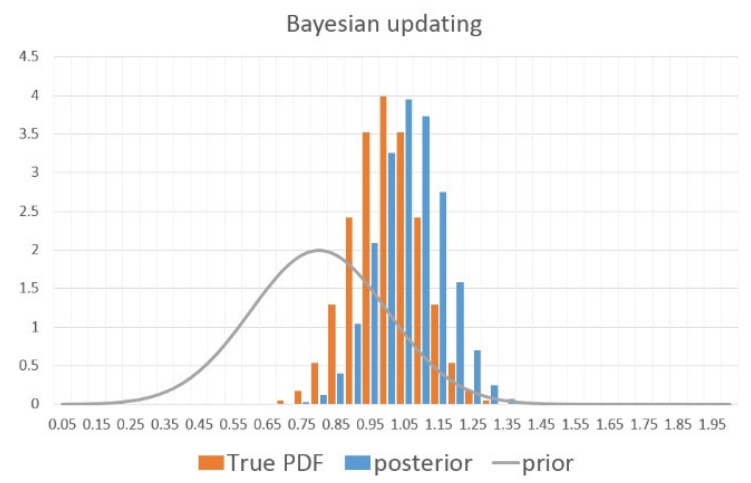

(f)

Figure 10. Bayesian updating at the stochastic field of the correlation distance $d=10.0$ : (a) How to perform Bayesian updating; (b) Observation point 1; (c) Observation point 2; (d) Observation point 3; (e) Observation point 4; (f) Observation point 5. 


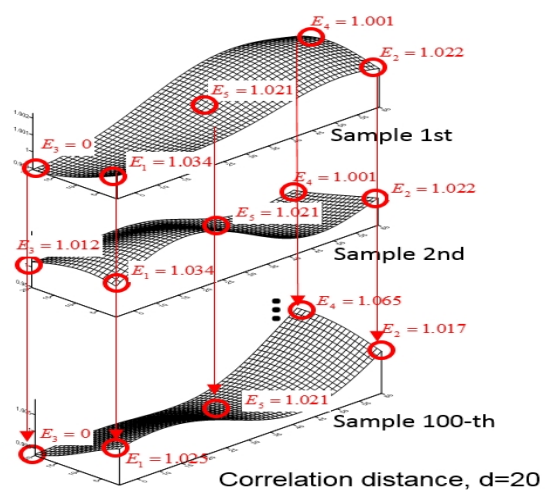

(a)

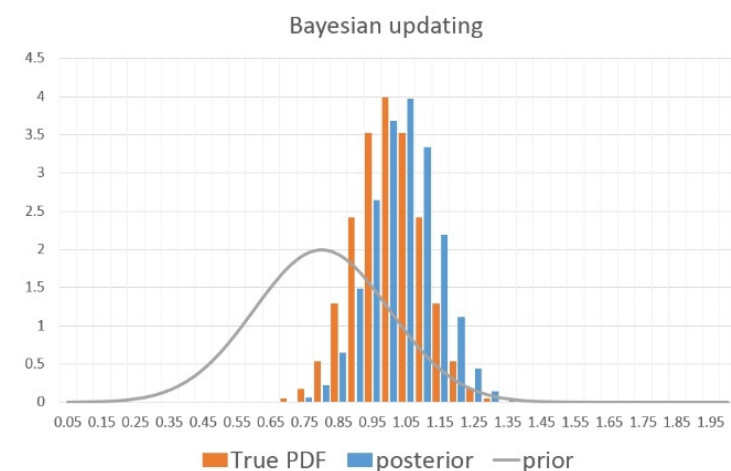

(c)

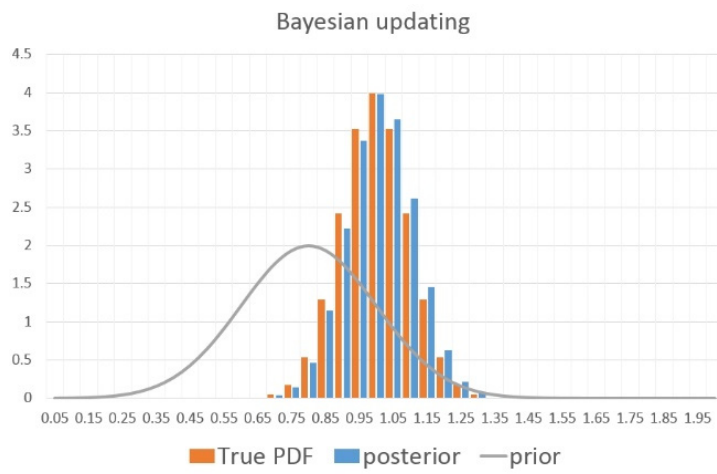

(e)

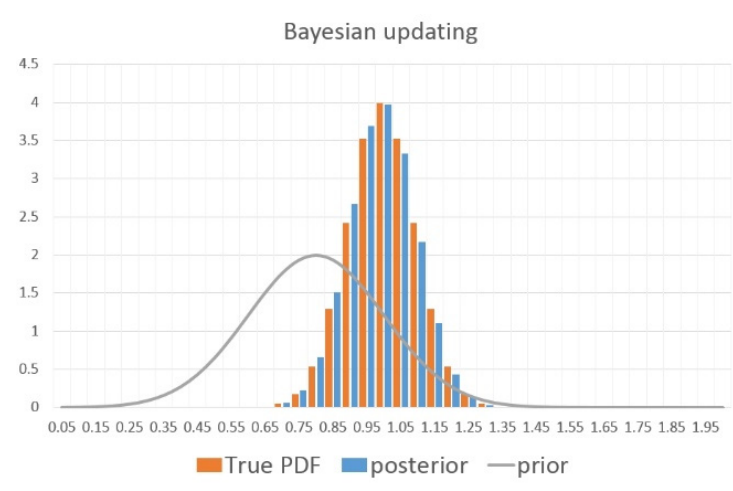

(b)

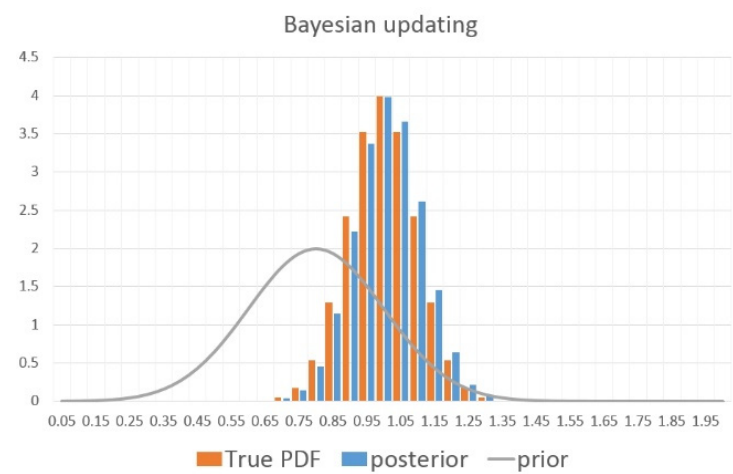

(d)

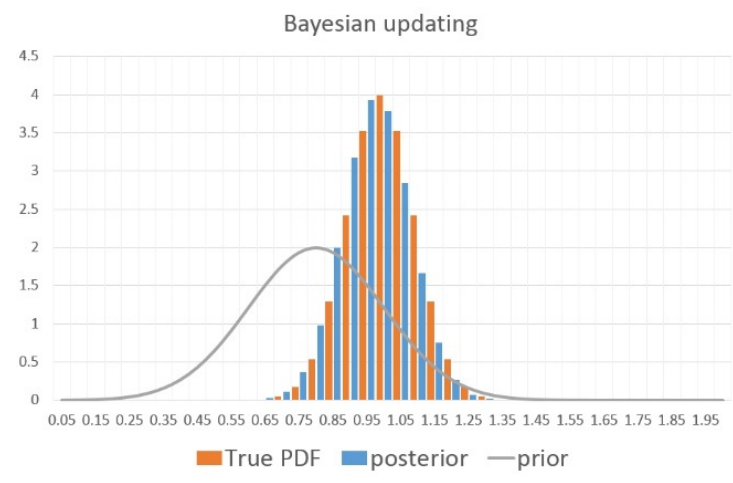

(f)

Figure 11. Bayesian updating at the stochastic field of the correlation distance $d=20.0$ : (a) How to perform Bayesian updating; (b) Observation point 1; (c) Observation point 2; (d) Observation point 3; (e) Observation point 4 ; (f) Observation point 5. 


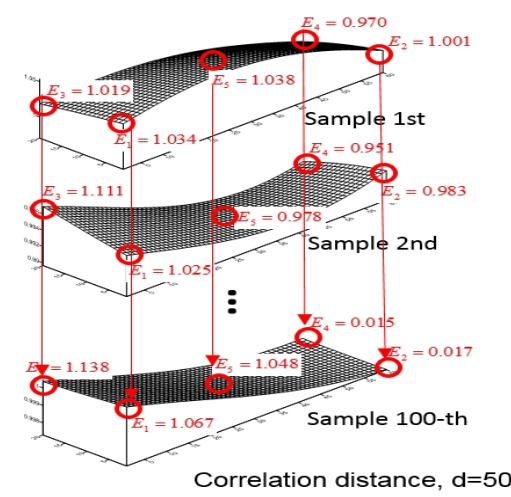

(a)

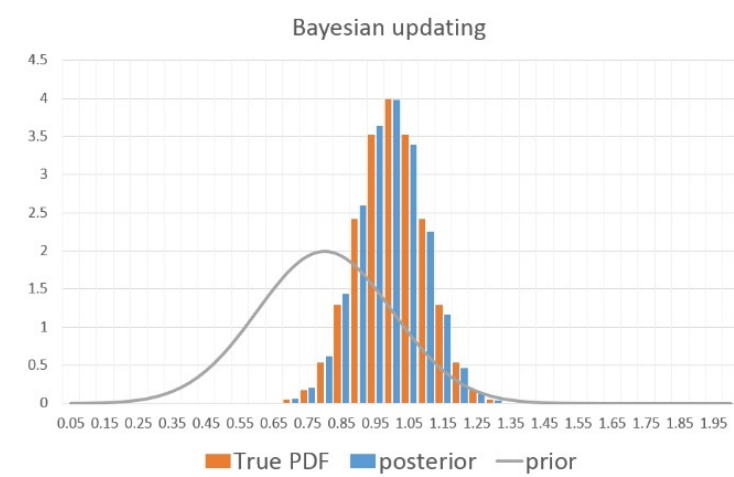

(c)

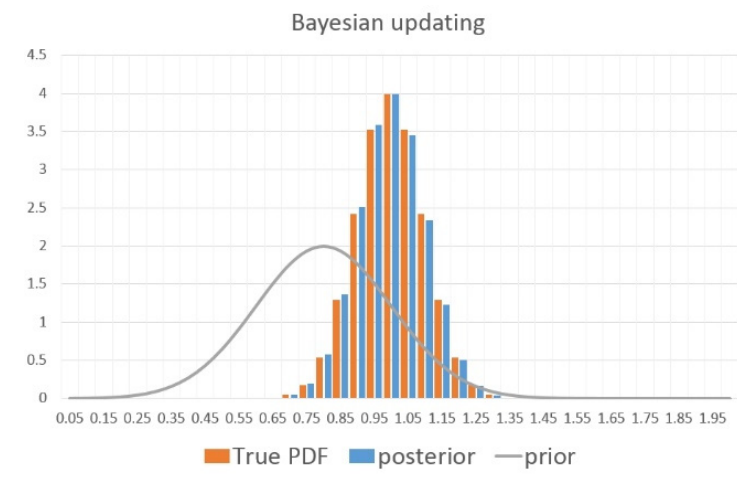

(e)

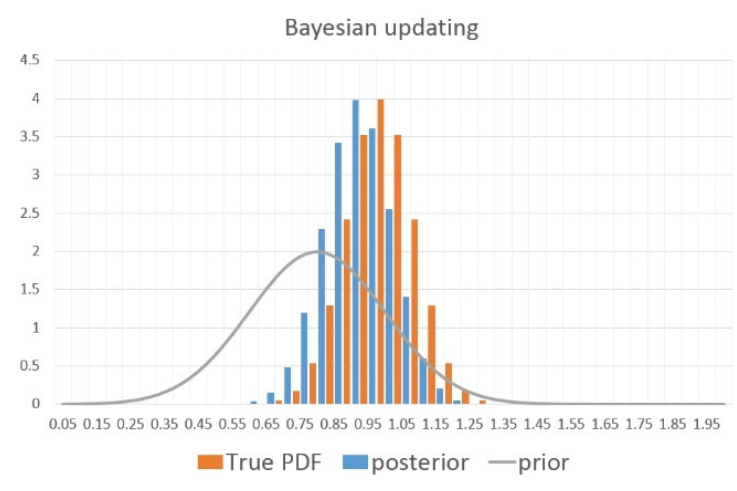

(b)

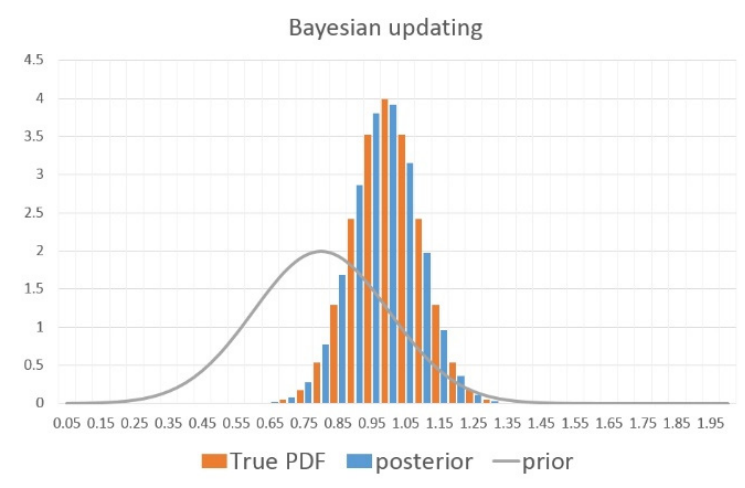

(d)

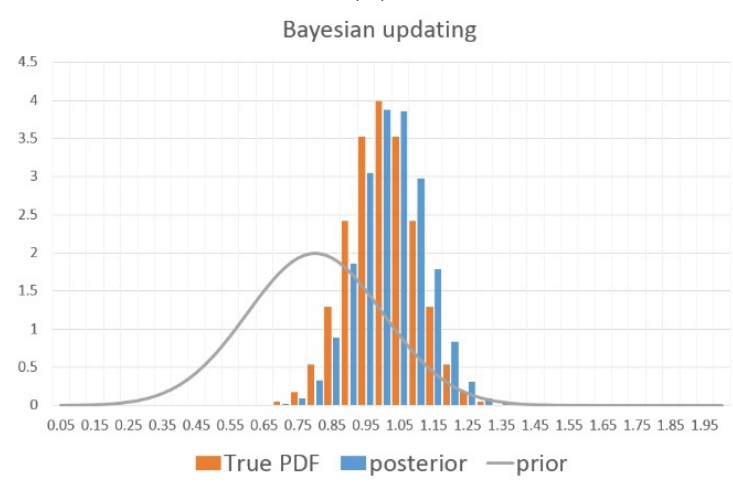

(f)

Figure 12. Bayesian updating at the stochastic field of the correlation distance $d=50.0$ : (a) How to perform Bayesian updating; (b) Observation point 1; (c) Observation point 2; (d) Observation point 3; (e) Observation point 4; (f) Observation point 5.

The elastic modulus values of the observation points (1 to 5) were updated using Bayes' theorem in a direction orthogonal to the stochastic plane. Bayesian updated results for observation points one through five are shown in subimages (b) through (f). The updated posterior probability was similar to the actual value at each position.

Figure 13 shows the comparison between the actual correlation distance and the estimated correlation distance at observation points one to five. Although the errors were different according to the observation positions from (a) to (e), the values achieved between the estimated correlation distance and the actual material showed good agreement. Notably, as the correlation distance (material homogeneity) increased, the correlation distance was accurately detected. 


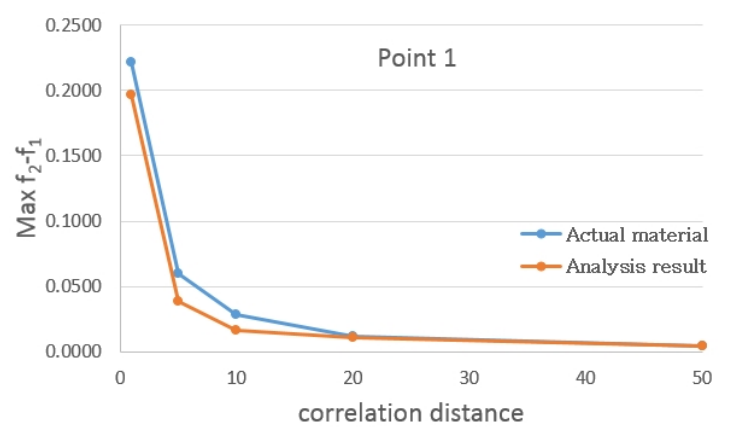

(a)

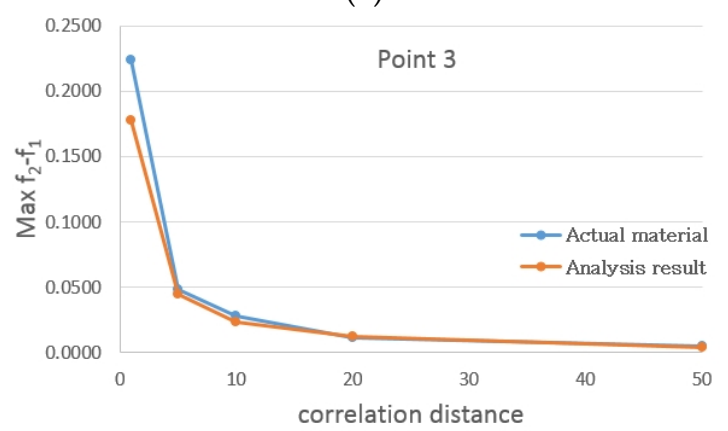

(c)

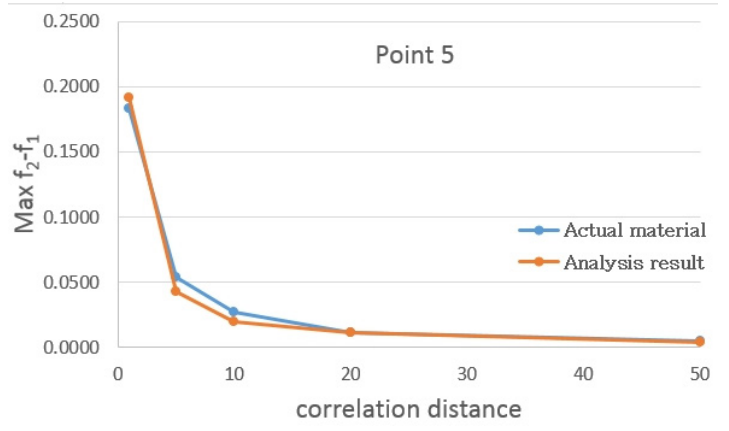

(e)

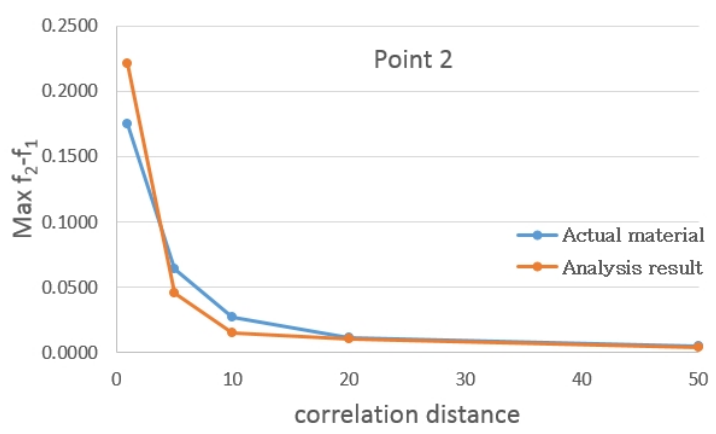

(b)

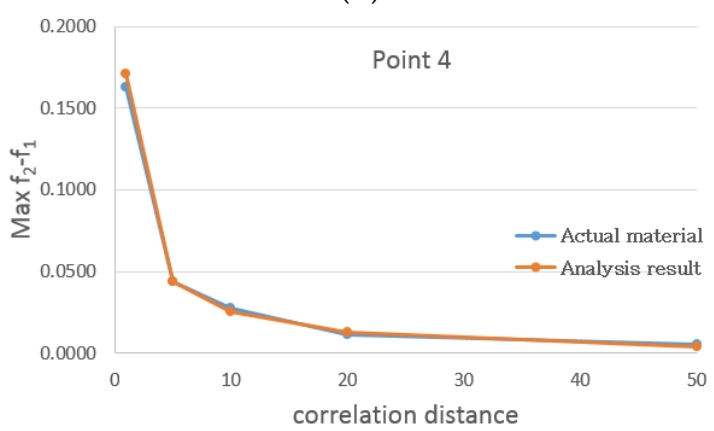

(d)

Figure 13. Comparison of $d$ for simulated (imitated) material and inversely analyzed $d:$ (a) Observation point 1; (b) Observation point 2; (c) Observation point 3; (d) Observation point 4; (e) Observation point 5 .

\section{Conclusions}

The existing design and analysis were based on the deterministic assumption that the design variables had the same value (=constant field) in the structural domain. However, many factors (Poisson's ratio, thickness, etc.) of the actual structure possess temporal and spatial randomness, and the material's elastic modulus contains uncertainties. The results can differ depending on the correlation distance as in the response variance. If the value $d$ in the actual material is not $d=\infty$, the result will be an under or overestimation.

In this study, we proposed a method of inversely estimating the correlation distance coefficient, which indicates the degree of uncertainty of the material, using the measured value of the observation data and the distance between measurement points. Probability characteristics and spatial randomness of the actual material can be estimated using some data only.

The concluding remarks of this paper can be summarized as follows:

- $\quad$ A 2D-1V sample data field was generated using a spectral representation method;

- Correlation distance estimation was performed using only partial values of sample data fields; and

- A new stochastic field was generated that considers the Bayesian updated partial values. 
The current probabilistic analysis can simulate spatial randomness through the correlation distance, but it does not know the spatial randomness of the actual material. Therefore, the present results are only assumptions, and their meaning is not conclusive.

In this study, a method of inversely estimating the correlation distance was proposed using measurement (observation), and it was confirmed that the actual correlation distance (d) agreed well with the estimated actual distance. Therefore, we could overcome the current disadvantage and find the true result of the actual structure, and not the assumption. Incidentally, the proposed method has a temporal advantage over the response variance. Verification was performed using an example by assuming that the program running time was $1 \mathrm{~min}$ at a time. A total of $100,000(=1000 \times 1 \times 100)$ minutes of program running time is required in order to analyze 1000 samples per correlation distance for COV from $\mathrm{d}=1 \sim 100$. This means a decrease in the number of interpretations of the Monte-Carlo simulation, and the program running time was reduced to a total of $1000(=1000 \times 1 \times 1)$ minutes.

Future studies need to be conducted considering the application of Poisson's ratio, cross-section (A), thickness ( $\mathrm{t}$ ), and uncertainty factors. Efforts to increase the accuracy of the estimates and actual values are also required in future study.

Author Contributions: Conceptualization, D.-Y.K., P.S., K.A. and S.-Y.C.; Methodology, D.-Y.K. and S.-Y.C.; Software, D.-Y.K. and S.-Y.C.; Validation, D.-Y.K., P.S., K.A. and S.-Y.C.; Formal analysis, D.-Y.K. and S.-Y.C.; Investigation, D.-Y.K. and S.-Y.C.; Resources, S.-Y.C., P.S. and K.A.; Data curation D.-Y.K. and S.-Y.C.; Writing—original draft preparation, D.-Y.K., S.-Y.C., P.S. and K.A.; Writing—review and editing, D.-Y.K., P.S., K.A. and S.-Y.C.; Visualization, D.-Y.K. and S.-Y.C.; Supervision, D.-Y.K. and S.-Y.C.; Project administration, D.-Y.K. and S.-Y.C.; Funding acquisition, D.-Y.K., S.-Y.C. and K.A. All authors have read and agreed to the published version of the manuscript.

Funding: This project was received funding from the European Union's Horizon 2020 research and innovation program under the Marie Sklodowska-Curie Grant agreement no. 841592. Pawel Sikora is supported by the Foundation for Polish Science.

Acknowledgments: The authors would like to acknowledge that this work was supported by the Korea Agency for Infrastructure Technology Advancement (KAIA) and the grant was funded by the Ministry of Land, Infrastructure and Transport (Grant 13IFIP-C113546-01 and Grant 20NANO-B156177-01).

Conflicts of Interest: The authors declare no conflicts of interest.

\section{References}

1. Chung, S.-Y.; Abd Elrahman, M.; Kim, J.-S.; Han, T.-S.; Stephan, D.; Sikora, P. Comparison of lightweight aggregate and foamed concrete with the same density level using image-based characterizations. Constr. Build. Mater. 2019, 211, 988-999. [CrossRef]

2. Pieralisi, R.; Cavalaro, S.H.P.; Aguado, A. Advanced numerical assessment of the permeability of pervious concrete. Cement Concr. Res. 2017, 102, 149-160. [CrossRef]

3. Janus, M.; Zajac, K. Concretes with photocatalytic activity. In High Performance Concrete Technology and Applications; Chapter 7; Yilmaz, S., Ed.; InTech: London, UK; pp. 141-160.

4. Janus, M.; Madraszewski, S.; Zajac, K.; Kusiak-Nejman, E.; Morawski, A.W.; Stephan, D. Photocatalytic activity and mechanical properties of cements modified with $\mathrm{TiO}_{2} / \mathrm{N}$. Materials 2019, 12, 3756. [CrossRef] [PubMed]

5. Schueller, G.I. Developments in Stochastic Structural Methods. Arch. Appl. Mech. 2006, 75, 755-773. [CrossRef]

6. Nguyen, V.T.; Noh, H.-C. The Stochastic Finite Element in the Natural Frequency of Functionally Graded Material Beam; Open Access Books-InthchOpen: London, UK, 2019.

7. Mena, J.D.A.; Margetts, L.; Mummery, P. Practical Application of the Stochastic Finite Element Method. Arch. Comput. Methods Eng. 2014, 23, 171-190. [CrossRef]

8. Joint Committee on Structural Safety (JCSS). Probabilistic Assessment of Existing Structures; RILEM Publications, S.A.R.L., Ed.; The Publishing Company of RILEM: Paris, France, 2001.

9. Wang, W.P.; Chen, G.H.; Yang, D.X.; Kang, Z. Stochastic isogeometric analysis method for plate structures with random uncertainty. Comput. Aided Geom. Design 2019, 74, 101772. [CrossRef] 
10. Choi, C.-K.; Noh, H.-C. Stochastic Finite Element Analysis of Plate Structures by Weighted Integral Method. Struct. Eng. Mech. 1996, 6, 703-715. [CrossRef]

11. Noh, H.-C. A Formulation for Stochastic Finite Element Analysis of Plate Structures with Uncertain Poisson's Ratio. Comput. Methods Appl. Mech. Eng. 2004, 193, 4857-4873. [CrossRef]

12. Kiureghian, A.D.; Ke, J.-B. The Stochastic Finite Element Method in Structural Reliability. Probabilistic Eng. Mech. 1988, 3, 83-91. [CrossRef]

13. Contreras, H. The Stochastic Finite-Element method. Comput. Struct. 1980, 12, 341-348. [CrossRef]

14. Spanos, P.D.; Beer, M.; Red-Horse, J. Karhunen-Loeve Expansion of Stochastic Processes with a Modified Exponential Covariance Kernel. J. Eng. Mech. ASCE 2007, 133, 773-779. [CrossRef]

15. Ghanem, R.; Spanos, P.D. Polynomial Chaos in Stochastic Finite Element. J. Appl. Mech. Trans. ASEM 1990, 57, 197-202. [CrossRef]

16. Kaminski, M. Generalized Perturbation-based Stochastic Finite Element Method in Elastostatics. Comput. Struct. 2007, 85, 586-594. [CrossRef]

17. Antonio, C.C.; Hoffbauer, L.N. Uncertainty Analysis Based on Sensitivity Applied to Angle-ply Composite Structures. Reliab. Eng. Syst. 2007, 92, 1353-1362. [CrossRef]

18. Deodatis, G. Weight Integral Method I: Stochastic Stiffness Matrix. J. Eng. Mech. 1991, 117, $1851-1864$. [CrossRef]

19. Lal, A.; Signh, B.N.; Kumar, R. Natural Frequency of Laminated Composite Plate Resting on an Elastic Foundation with Uncertain System Properties. Struct. Eng. Mech. 2007, 27, 199-222. [CrossRef]

20. Lawrence, M.A. Basis Random Variables in Finite Element Analysis. Int. J. Numer. Methods Eng. 1987, 24, 1849-1863. [CrossRef]

21. Ngah, M.F.; Young, A. Application of the Spectral Stochastic Finite Element Method for Performance Prediction of Composite Structures. Compos. Struct. 2007, 78, 447-456. [CrossRef]

22. Papadopoulos, V.; Deodatis, G.; Papadrakakis, M. Flexibility-based Upper Bounds on the Response Variability of Simple Beams. Comput. Method Appl. Mech. Eng. 2005, 194, 1385-1404. [CrossRef]

23. Micaletti, R.C. Direct Generation of Non-Gaussian weighted Integrals. J. Eng. Mech. ASCE 2000, 126, 66-75. [CrossRef]

24. Deodatis, G.; Micaletti, R.C. Simulation of Highly Skewed Non-Gaussian Stochastic Processes. J. Eng. Mech. ASCE 2001, 126, 66-75. [CrossRef]

25. Deodatis, G.; Graham, B.L. A Hierarchy of Upper Bounds on the Response of Stochastic System with Large Variation of Their Properties. Probabilistic Eng. Mech. 2003, 18, 349-363. [CrossRef]

26. Noh, H.-C. Stochastic Finite Element analysis of Composite Plates Considering Spatial Randomness of Material Properties and Their Correlations. Steel Compos. Struct. 2011, 11, 115-130. [CrossRef]

27. Noh, H.-C. Stochastic Behavior of Mindlin plate with uncertain geometrical and material parameters. Steel Probabilistic Eng. Mech. 2005, 20, 296-306. [CrossRef]

28. Shinozuka, M.; Deodatis, G. Simulation of Stochastic Processes by Spectral Representation. Am. Soc. Mech. Eng. 1991, 44, 191-204. [CrossRef]

29. Shinozuka, M.; Deodatis, G. Simulation of Multi-Dimensional Gaussian Stochastic Fields by Spectral Representation. Am. Soc. Mech. Eng. 1996, 49, 29-53. [CrossRef]

30. Congdon, P. The Bayesian Method: Its benefits and implementation. In Bayesian Statistical Modelling; John Wiley \& Sons: Chichester, West Sussex, UK, 2006; pp. 1-18.

31. Carmer, H.; Leadbetter, M. Stationary and Related Stochastic processes. Soc. Ind. Appl. Math. 1967, 10, 94-95.

32. Svetlisky, V.A. Stationary Random Function (Processes); Springer: Berlin/Heidelberg, Germany, 1962; pp. 77-100.

33. Shinozuka, M.; Deodatis, G. Response Variability of Stochastic Finite Element System. J. Eng. Mech. ASCE 1988, 114, 499-519. [CrossRef]

34. Shinozuka, M. Monte Carlo Solution of Structural Dynamics. Comput. Struct. 1972, 2, 855-874. [CrossRef]

35. Ta, D.H.; Nguyen, D.H.; Nguyen, T.K.; Noh, H.-C. The Variability of Dynamic Responses of Beams Resting on Elastic Foundation Subjected to Vehicle With Random System Parameters. Appl. Math. Model. 2019, 67, 676-687.

36. Ta, D.H.; Lam, N.N. Investigation into the Effect of Random Load on the Variability of Response of Plate by Using Monte Carlo Simulation. Int. J. Civil Eng. Technol. (IJCIET) 2016, 7, 169-176.

37. Nguyen, H.X.; Ta, D.H.; Lee, J.-H.; Nguyen-Xuan, H. Stochastic buckling behavior of laminated composite structures with uncertain material properties. Aerosp. Sci. Technol. 2017, 66, 274-283. [CrossRef] 
38. An, D.; Won, J.H.; Kim, E.J.; Choi, J.H. Reliability Analysis Under Input Variable and Metamodel Uncertainty Using Simulation Method Based on Bayesian Approach. Trans. Korean Soc. Mech. Eng. A. 2009, 33, 1163-1170. [CrossRef]

39. Jordan, M.; Kleinberg, M.; Schölkopf, B. Information Science and Statistics: Its benefits and implementation. In Bayesian Probabilities; Springer Science: New York, NY, USA, 2006; pp. 24-28.

40. Wei, Z.; Conlon, E.M. Parallel Markov chain Monte Carlo for Bayesian Hierarchical Models with Big Data. in Two Stage. J. Appl. Stat. 2019, 46, 1917-1936. [CrossRef]

41. Andrew, G.; John, B.C.; Hal, S.S.; David, B.D.; Aki, V.; Donald, B.R. Bayesian Data Analysis, 3rd ed.; Taylor \& Francis Group: Abingdon, UK, 2013; pp. 29-69.

42. Nguyen, V.T.; Noh, H.-C. Investigation Into the Effect of Random Material Properties on the Variability of Natural Frequency of Functionally Graded Beam. KSCE J. Civil Eng. 2017, 21, 1264-1272.

(C) 2020 by the authors. Licensee MDPI, Basel, Switzerland. This article is an open access article distributed under the terms and conditions of the Creative Commons Attribution (CC BY) license (http://creativecommons.org/licenses/by/4.0/). 